\title{
BRCA1-positiv: Stillen schützt vor Brustkrebs
}

\author{
Stillen senkt das Brustkrebsrisiko, auch bei genetischer Disposition durch BRCA1-, nicht aber bei BRCA2-Mutationen. \\ Das ergab die bisher größte Studie zum Einfluss des Stillens auf $B R C A$-assoziierte Mammakarzinome.
}

$\mathrm{D}$ as hatte eine im Jahr 2004 veröffentliche Fall-Kontroll-Studie der „Hereditary Breast Cancer Clinical Study Group“ gezeigt. Mit mittlerweile 1.665 Frauen mit invasivem Mammakarzinom und ebenso vielen brustgesunden Frauen hat die kanadische Studiengruppe nun ihre Ergebnisse aktualisiert. 1.243 Paarungen hatten eine Mutation in BRCA1, die übrigen in BRCA2. Fälle und Kontrollen waren identisch bei Geburtsjahr, $\mathrm{Mu}$ tationsstatus und Zahl der Geburten.

$B R C A 1$-Trägerinnen mit Brustkrebs hatten kürzer gestillt als die gesunden Kontrollen (8,8 Monate vs. 10,4 Monate; $\mathrm{p}=0,0009$ ). Je länger die Stillzeiten, desto geringer das Krebsrisiko: Nach mindestens einem Jahr war es um $32 \%$, nach mindestens zwei Jahren um $49 \%$ reduziert. Das entsprach einer Risikominderung um $19 \%$ pro Stilljahr. Zum Vergleich: In der Allgemeinbevölkerung wird das (insgesamt niedrigere) Risiko laut einer Metaanalyse dadurch nur um 4,3\% gesenkt. Bei den BRCA2-Trägerinnen beeinflusste das Stillen das Risiko nicht.

Für die inverse Beziehung zwischen Stillen und Mammakarzinomrisiko kommen verschiedene Mechanismen infrage, v. a. die veränderten Hormonspiegel (weniger Östrogen und Progesteron, mehr Prolaktin). Ebenso könnte die Sekretion von Östrogenen und Karzinogenen aus den Drüsengängen sowie die Differenzierung des Brustgewebes eine Rolle spielen. Die Autoren vermuten, dass das Ausmaß der Östrogen-Exposition bei BRCA1Trägerinnen mit Brustkrebs korreliert. Dazu würde passen, dass außer dem Stillen auch eine späte Menarche und die bilaterale Salpingo-Oophorektomie protektiv wirken. Frauen mit einer BRCA1Mutation, so die Autoren, sollten deswegen über den Nutzen des Stillens hinsichtlich ihres Brustkrebsrisikos aufgeklärt werden.

Dr. Beate Schumacher

Kotsopoulos J et al. Breastfeeding and the risk of breast cancer in BRCA1 and BRCA2 mutation carriers. Breast Cancer Res. 2012; 14(2):R42. [Epub ahead of print].
Kommentar: Die Identifikation krebspräventiver Faktoren ist ein zentrales Anliegen der Onkologie, insbesondere für Menschen mit einem gegenüber der Allgemeinbevölkerung deutlich erhöhten Krebsrisiko. Bei Frauen mit einer BRCA1- und BRCA2-Mutation liegt das Lebenszeitrisiko für Brustkrebs bei ca. $80 \%$. In den nicht-direktiven Beratungsgesprächen werden gesunde und erkrankte Frauen mit erhöhtem familiärem Brustkrebsrisiko bzw. Mutationsträgerinnen über Maßnahmen der primären (z. B. prophylaktische Operationen) und der sekundären Prävention (Teilnahme an intensivierten Früherkennungsuntersuchungen in spezialisierten Zentren) informiert. So kann eine prophylaktische Mastektomie das Brustkrebsrisiko einer BRCA-Mutationsträgerinnen auf ca. $5 \%$ senken [Meindl $A$ et al. Dtsch Arztebl Int. 2011; 108(19):323-30].

Nun stellt sich die Frage, ob die Ergebnisse der Arbeit von Kotsopoulos et al. Eingang in die Beratung von BRCA-Mutationsträgerinnen finden sollten. Die Fall-KontrollStudie weist zum einen Mängel bei der Vergleichbarkeit der beiden Kollektive auf. So setzte z. B. bei den erkrankten BRCA1Mutationsträgerinnen die Menarche signifikant früher ein als bei den gesunden Mutationsträgerinnen. Der Menarche-Zeitpunkt ist für Frauen der Allgemeinbevölkerung ein bekannter Risikofaktor für ein Mammakarzinom. Auch haben Mutationsträgerinnen mit Mammakarzinom signifikant seltener Hormone zu sich genommen als gesunde Mutationsträgerinnen ( 5 vs. $12 \% ; p<0,0001)$ und signifikant kürzer gestillt ( 8,8 vs. 10,4 Monate; $p=0,0009)$. Insgesamt wird in der Studie der Effekt einer Stillzeit von $\geq 1$ Jahr angegeben. Der Effekt realistischer Stillzeiten heutiger junger Mütter $<1$ Jahr wurde nicht berechnet.

Ein grundsätzlich limitierender Faktor bei retrospektiven Selbstauskünften von Patientinnen ist die "Erinnerungsverzerrung". Ungenaue Angaben z. B. über Länge der Stillperiode oder alleiniges Stillen versus Stillen und Zufüttern oder ein Wahrnehmen des Stillens als Risikofaktor für Brustkrebs und eine damit einhergehende überdeutliche Erinnerung daran können zu einem fälschlichen Zusammenhang von (Risiko-) Faktoren und Brustkrebsentstehung führen.

Erschwerend für die Interpretation der Daten ist, dass zu relativen Risikozahlen wie einer 32\%igen Risikoreduktion für stillende BRCA1-Mutationsträgerinnen nicht gesagt wird, über welchen Zeitraum dieser potenziell schützende Effekt beobachtet wird.

Interessant wäre auch ein Vergleich des Brustkrebsrisikos von Nulliparae versus Parae ohne bzw. mit Stillen. In der vorliegenden Studie wurde das Mammakarzinomrisiko bei Nulliparae durch Fokussierung auf die Frage nach dem Einfluss des Stillens auf das Krebsrisiko von der Analyse ausgeschlossen. Aber es gibt in der Literatur Hinweise auf ein z.T. in Abhängigkeit vom Alter erniedrigtes Brustkrebsrisiko für Erstgebärende mit BRCA1/2-Mutationen im Vergleich zu Parae [z. B. Cullinane CA et al. Int J Cancer. 2005;117(6):988-91; Andrieu N et al. J Natl Cancer Inst. 2006;98(8):535-44], wenn auch insgesamt die Datenlage zum Zusammenhang von Parität und Brustkrebsrisiko bei

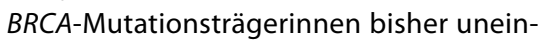
heitlich ist.

Möglich ist auch, dass Mutationsträgerinnen aufgrund der erschwerten Diagnose eines Mammakarzinoms während der Stillzeit einen scheinbaren Vorteil vom Stillen haben, weil die Diagnose des Mammakarzinoms entsprechend verzögert erfolgte.

Die Arbeit von Kotsopoulos ist interessant, weist aber einige Mängel auf. Auch lässt sich die Empfehlung der Autoren zu einer womöglich 2-bis 3-jährigen Stillzeit wohl kaum in die Lebenswirklichkeit heutiger junger Mütter integrieren. Um belastbare Aussagen zum Effekt des Stillens auf das Brustkrebsrisiko von Mutationsträgerinnen zu erhalten, muss man prospektiv randomisierte Daten abwarten, wie sie z.B. derzeit im Deutschen Konsortium für Familiären Brust- und Eierstockkrebs erfasst werden.

PD Dr. Kerstin Rhiem 